\title{
Quantum Mechanical Resonance and Limiting Absorption: The Many Body Problem
}

\author{
Andreas Orth* \\ Zentralabteilung für Informatik und Kommunikation der Hoechst A.G., \\ D-6000 Frankfurt, Federal Republic of Germany
}

\begin{abstract}
We introduce a notion of quantum mechanical resonance that does not rely on analytic continuation of resolvent or scattering matrix and relate it to slow temporal decay of certain distinguished resonant states. We proceed to prove existence of resonances for the generalized many body Schrödinger operator for a rather large class of potentials containing Coulomb and Yukawa, but also nonsymmetric and nonanalytic potentials with Coulomblike singularities at the origin and certain differentiability and decay properties.
\end{abstract}

\section{Introduction}

There are several instances in classical quantum mechanics where a system possesses quasi-stable states, that are not eigenstates of the associated Schrödinger operator. For these states ordinary spectral and scattering theory fails to explain the longevity of these states

There have been several approaches proposed in the last fifteen years or so to account for these states and to give estimates of their half-lives. The most notable among these involve continuing the resolvent of the Schrödinger operator across the real axis onto the second sheet of the complex plane and identifying poles of this continued resolvent. We refer in particular to Simon [S1] and to some comments in Sect. 2 of this paper.

Such poles are called resonances and can be made responsible for

- longevity of associated quasi-eigenstates

- peaks in the spectral density of the operator

- peaks in the associated scattering amplitude.

In this paper we propose to study resonances based on limting absorption principles applied to the Schrödinger operator. This means we identify resonances directly as isolated peaks in the spectral density function and proceed to make the

\footnotetext{
* Most of this work was done while the author was at Fachbereich Mathematik der Universität Frankfurt, D-6000 Frankfurt, Federal Republic of Germany
} 
connection to slow time decay. We make no mention of scattering matrix here, but refer to a discussion on the relation between Livsic' matrix (or energy dependent Hamiltonian) and the scattering matrix by Howland in [Ho].

The advantage for our technique is that restrictions on the potentials involved are less strict. In particular, for the many body problem, which is treated in Sect. 3, we need no analyticity or symmetry assumptions at all! Decay and differentiability conditions suffice, hence the study of resonances is placed closer to many other problems in spectral and scattering theory, such as existence and completeness of wave operators, absence of singular continuous spectrum etc.

In the first section we state our definition of resonance and relate it to temporal decay of the associated resonance state. In section two we show how some of the existing notions of resonance can be compared to ours. Finally, in section three we treat the many body Schrödinger operator and identify resonances for a very general class of potentials. In a follow-up paper [OP] we have treated the two body Stark-problem of perturbation by a homogeneous field.

\section{Resonances}

Our main instrument in treating resonances will be the Livsic matrix (cf. Howland [Ho]). For a self adjoint operator $H$ in the Hilbert space $\mathscr{H}$ and the finite dimensional subspace $K$ in $D(H)$, the domain of $H$, we define it as follows. Let $P$ be the orthogonal projection onto $K, \bar{P}=I-P, \bar{H}=\bar{P} H \bar{P}$, and $E$ the spectral resolution of $H$.

Definition 1.4. We call $B(z)=P H P-P H \bar{P}(\bar{H}-z)^{-1} \bar{P} H P$, initially defined for $\operatorname{Im} z \neq 0$, the Livsic matrix of $H$ and $K$.

Theorem 1.2. The Livsic matrix has the following fundamental properties:

(i) (defining property) $P(H-z)^{-1} P=(B(z)-z)^{-1}$.

(ii) (dissipativity) For $\operatorname{Im} z>0, \operatorname{Im} B(z)=(2 i)^{-1}\left(B(z)-B(z)^{*}\right) \leqq 0$.

(iii) (relation to $E$ ) Let $B(z)$ have a continuous extension onto a real interval $I$, and let $J \subset I$ be such that no $\lambda \in J$ is an eigenvalue of $B(\lambda)=\lim _{\varepsilon \rightarrow 0} B(\lambda+i \varepsilon)$, then

$$
P E(J) P=\pi^{-1} \int_{J} \operatorname{Im}(B(\lambda)-\lambda)^{-1} d \lambda .
$$

If $\lambda \in I$ is an eigenvalue of $B(\lambda)$, then

$$
P E(\{\lambda\}) P=\lim _{\varepsilon \rightarrow 0} \varepsilon \operatorname{Im}(B(\lambda+i \varepsilon)-\lambda-i \varepsilon)^{-1} .
$$

Proof. (i) is seen by writing $H$ as a perturbation of its diagonalisation with respect to $P$, twice using the resolvent equation, then projecting and bootstrapping. (ii) and (iii) are left to the reader.

Remark. Notice that since $P$ and $(H-z)^{-1}$ need not commute, $(B(z)-z)^{-1}$ is not generally normal.

Condition 1.3. Let $H_{0}$ be self adjoint in $\mathscr{H}, W$ closed and symmetric, such that $D\left(H_{0}\right) \cap D(W)$ is dense in $\mathscr{H}$. For small $\kappa$, let $H_{0}+\kappa W \subset H(\kappa)$ be essentially self 
adjoint on $D\left(H_{0}\right) \cap D(W)$ and self adjoint on $D(H(\kappa))$. (This implies strong resolvent continuity of $H(\kappa)$.)

Let $\lambda_{0}$ be an eigenvalue of finite degeneracy $n$ of $H_{0}$, and $K$ the associated eigenspace. Define $\bar{P}=I-P, \bar{H}(\kappa)=\bar{P} H(\kappa)=\bar{P} H(\kappa) \bar{P}, E_{\kappa}$ as the spectral resolution of $H(\kappa)$. Let $\Lambda=P W P$ and $f(z, \kappa)=P W \bar{P}(\bar{H}(\kappa)-z)^{-1} \bar{P} W P ; \Lambda$ is symmetric, let $\lambda_{1}, \ldots, \lambda_{n}$ be its eigenvalues.

For $\operatorname{Im} z>0 \operatorname{Im} f(z, \kappa) \geqq 0$, and the Livsic matrix for $H(\kappa)$ and $K$ is

$$
B(z, \kappa)=\lambda_{0}+\kappa \Lambda-\kappa^{2} f(z, \kappa) .
$$

In particular, if $f(z, \kappa)$ can be extended onto a real interval $I$, then so can $B(z, \kappa)$, and Theorem 1.2(iii) applies.

Definition 1.4. We assume Condition 1.3 and define:

The operator family $H(\kappa)$ has a simple resonance at $\lambda_{0}$, if $\lambda_{0}$ is nondegenerate and if there are

- a real neighbourhood $I$ of $\lambda_{0}$,

- a real neighbourhood $U$ of 0 ,

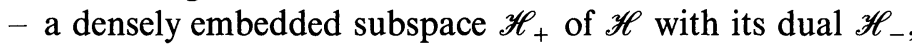
such that

(i) for $\kappa \in U,(\bar{H}(\kappa)-z)^{-1}$ has a continuous extension from $\mathbb{C} / \mathbb{R}$ onto $z \in I$ as an operator in $B\left(\mathscr{H}_{+}, \mathscr{H}_{-}\right)$. This continuation is Lipschitz-continuous with constant $L(\kappa)=o\left(\kappa^{-2}\right)$.

(ii) $K \subset \mathscr{H}_{+}$, and $W(K) \subset \mathscr{H}_{+}$.

(iii) For $\kappa \in U$ and all possible eigenvalues $\mu(\kappa) \in I$ of $H(\kappa)$, the associated eigenvectors are in $\mathscr{H}_{+}$.

Remark. (i) We have given a similar definition in [Or], however (iii) is new. It allows us to show, that no "unwanted" eigenvalues can exist in the vicinity of $I$. This question was not addressed in [Or].

(ii) Condition (i) is made in order that $\kappa^{2} f(z, \kappa)$ is Lipschitz-continuous in $z$, with constant going to zero as $\kappa \rightarrow 0$.

(iii) For Theorems 1.5 and 1.8 Lipschitz-continuity in (i) can be replaced by nondegeneracy of $\operatorname{Im} f\left(\lambda_{0}, 0\right)$. In this case $\lambda(\kappa)$ as defined in the following theorem can be replaced by simply $\lambda_{0}$.

Theorem 1.5 (spectral concentration). Let $H(\kappa)$ have a simple resonance at $\lambda_{0}$, let $\lambda(\kappa)$ be the unique solution of the fixed point equation

$$
\lambda(\kappa)=\operatorname{Re} B(\lambda(\kappa), \kappa),
$$

let $B(\kappa)=B(\lambda(\kappa), \kappa), \Gamma(\kappa)=-\operatorname{Im} B(\kappa)$. Now choose $\delta(\kappa) \geqq 0$, such that for $\Gamma(\kappa)=0$, $\delta(\kappa)=0$, while for $\Gamma(\kappa) \neq 0$ and $\kappa \rightarrow 0, \max \left(\delta(\kappa), \frac{\kappa^{2} L(\kappa) \delta(\kappa)}{\Gamma(\kappa)}\right) \rightarrow 0$, but at the same time $\frac{\Gamma(\kappa)}{\delta(\kappa)} \rightarrow 0$. Let $J(\kappa)=[\lambda(\kappa)-\delta(\kappa), \lambda(\kappa)+\delta(\kappa)]$. Then $E_{\kappa}(J(\kappa)) \stackrel{s}{\rightarrow} P$.

Proof. $\lambda(\kappa)$ is found using Banach's Fixed Point theorem and Lipschitz-continuity of $f$. Hence $B(\kappa), \Gamma(\kappa)$ are well defined, and $\delta(\kappa)$ and $J(\kappa)$ can be determined. Notice 
that $\Gamma(\kappa)=O\left(\kappa^{2}\right)$, hence $\delta(\kappa)=o\left(\min \left(L(\kappa)^{-1}, 1\right)\right)$. We prove $E_{\kappa}(J(\kappa)) \bar{P} \rightarrow 0$ : Let $\psi \in \mathscr{H}, \varepsilon>0$, then

$$
\left\|E_{\kappa}(J(\kappa)) \bar{P} \psi\right\| \leqq\left\|E_{\kappa}(J(\kappa)) E_{0}\left(\mathbb{R} \backslash\left(\lambda_{0}-\varepsilon, \lambda_{0}+\varepsilon\right)\right) \psi\right\|+\left\|E_{0}\left(\left(\lambda_{0}-\varepsilon, \lambda_{0}+\varepsilon\right)\right) \bar{P} \psi\right\| .
$$

Choosing a small $\varepsilon$ takes care of the second term, the first goes to zero as $\kappa \rightarrow 0$. We prove $E_{\kappa}(J(\kappa)) P \stackrel{s}{\rightarrow} P$ : Let $\varphi, \psi \in \mathscr{H}$ : For sufficiently small $\kappa, J(\kappa) \subset I$. Hence for $\kappa$, such that $\Gamma(\kappa) \neq 0$ :

$$
\begin{aligned}
\left\langle\varphi, E_{\kappa}(J(\kappa)) P \psi\right\rangle & =\left\langle P \varphi, E_{\kappa}(J(\kappa)) P \psi\right\rangle+o(1) \\
& =\pi^{-1} \int_{J(\kappa)}\left\langle P \varphi, \operatorname{Im}(B(\lambda, \kappa)-\lambda)^{-1} P \psi\right\rangle d \lambda+o(1) \\
& =\pi^{-1} \int_{\mathbb{R}}\left\langle P \varphi,(B(\kappa)-\lambda)^{-1} P \psi\right\rangle d \lambda+o(1)
\end{aligned}
$$

by Lemma 1.6 to be proven below;

$$
=\langle\varphi, P \psi\rangle+o(1) .
$$

For $\kappa$, such that $\Gamma(\kappa)=0: J(\kappa)=\{\lambda(\kappa)\}, B(\kappa)=\lambda(\kappa),|B(\lambda(\kappa)+i \varepsilon, \kappa)| \leqq \kappa^{2} L(\kappa) \varepsilon$, hence

$$
\begin{aligned}
\left\langle\varphi, E_{\kappa}(J(\kappa)) P \psi\right\rangle & =\left\langle P \varphi, E_{\kappa}\left(\left\{\lambda_{\kappa}\right\}\right) P \psi\right\rangle+o(1) \\
& =\lim _{\varepsilon \rightarrow \infty} \varepsilon \operatorname{Im}\left\langle P \varphi,(B(\lambda(\kappa)+i \varepsilon, \kappa)-\lambda(\kappa)-i \varepsilon)^{-1} P \psi\right\rangle+o(1) \\
& =\lim _{\varepsilon \rightarrow 0} \varepsilon \operatorname{Im}\left\langle P \varphi, i / \varepsilon(1+o(1))^{-1} P \psi\right\rangle+o(1)=\langle\varphi, P \psi\rangle+o(1) .
\end{aligned}
$$

This proves weak convergence. Strong convergence follows after setting $\varphi=P \psi$.

Lemma 1.6. For $\kappa$ such that $\Gamma(\kappa) \neq 0$ :

$$
\chi_{J(\kappa)}(\lambda) \operatorname{Im}(B(\lambda, \kappa)-\lambda)^{-1}-\operatorname{Im}(B(\kappa)-\lambda)^{-1} \rightarrow 0
$$

in the $L^{1}(\mathbb{R})$-norm with respect to $\lambda$ as $\kappa \rightarrow 0$.

Proof. Although both terms diverge, their difference converges, as can be seen by making the substitution

$$
\lambda^{\prime}=\frac{1}{\Gamma(\kappa)}(\lambda-\lambda(\kappa)), \quad \text { i.e. } \lambda=\lambda\left(\lambda^{\prime}, \kappa\right)=\lambda(\kappa)+\lambda^{\prime} \Gamma(\kappa) .
$$

Let $J^{\prime}(\kappa)=\frac{1}{\Gamma(\kappa)}(J(\kappa)-\lambda(\kappa))=\left[-\frac{\delta(\kappa)}{\Gamma(\kappa)}, \frac{\delta(\kappa)}{\Gamma(\kappa)}\right] \rightarrow \mathbb{R}$.

Statement (1.1) becomes equivalent to

$$
\chi_{J^{\prime}(\kappa)}\left(\lambda^{\prime}\right) \operatorname{Im}\left(B\left(\lambda\left(\lambda^{\prime}, \kappa\right), \kappa\right)-\lambda\left(\lambda^{\prime}, \kappa\right)\right)^{-1} \Gamma(\kappa)-\operatorname{Im}\left(B(\kappa)-\lambda\left(\lambda^{\prime}, \kappa\right)\right)^{-1} \Gamma(\kappa) \rightarrow 0
$$

in the $L^{1}$-norm with respect to $\lambda^{\prime}$ as $\kappa \rightarrow 0$.

Now fix $\lambda^{\prime} \in \mathbb{R}$, then for sufficiently small $\kappa, \lambda^{\prime} \in J^{\prime}(\kappa)$, i.e. $\left|\lambda\left(\lambda^{\prime}, \kappa\right)-\lambda(\kappa)\right| \leqq \delta(\kappa)$ and

$$
\left|B\left(\lambda\left(\lambda^{\prime}, \kappa\right), \kappa\right)-B(\kappa)\right| \leqq \kappa^{2} L(\kappa) \delta(\kappa)
$$


An indirect use of the resolvent equation yields

$$
\begin{aligned}
& \left(B\left(\lambda\left(\lambda^{\prime}, \kappa\right), \kappa\right)-\lambda\left(\lambda^{\prime}, \kappa\right)\right)^{-1} \\
& \quad=\left(B(\kappa)-\lambda\left(\lambda^{\prime}, \kappa\right)\right)^{-1}\left[I+\left(B\left(\lambda\left(\lambda^{\prime}, \kappa\right), \kappa\right)-B(\kappa)\right)\left(B(\kappa)-\lambda\left(\lambda^{\prime}, \kappa\right)\right)^{-1}\right]^{-1} .
\end{aligned}
$$

Using (1.3), $\left.\mid B(\kappa)-\lambda\left(\lambda^{\prime}, \kappa\right)\right)^{-1} \mid \leqq \Gamma(\kappa)^{-1}$ and the assumptions on $\delta(\kappa)$, we see that in (1.4) the expression in square brackets is invertible with a uniformly bounded inverse and it converges to 1 . Thus we have proven pointwise convergence of (1.2). Now

$$
\begin{aligned}
\operatorname{Im}\left(B\left(\lambda\left(\lambda^{\prime}, \kappa\right), \kappa\right)-\lambda\left(\lambda^{\prime}, \kappa\right)\right)^{-1}= & -\left(B\left(\lambda\left(\lambda^{\prime}, \kappa\right), \kappa\right)^{*}-\lambda\left(\lambda^{\prime}, \kappa\right)\right)^{-1} \\
& \times \operatorname{Im} B\left(\lambda\left(\lambda^{\prime}, \kappa\right), \kappa\right)\left(B\left(\lambda\left(\lambda^{\prime}, \kappa\right), \kappa\right)-\lambda\left(\lambda^{\prime}, \kappa\right)\right)^{-1} .
\end{aligned}
$$

By (1.3) $\left|\operatorname{Im} B\left(\lambda\left(\lambda^{\prime}, \kappa\right), \kappa\right)+\Gamma(\kappa)\right| \leqq \kappa^{2} L(\kappa) \delta(\kappa)$ and for sufficiently small $\kappa$, $\left|\operatorname{Im} B\left(\lambda\left(\lambda^{\prime}, \kappa\right), \kappa\right)\right| \leqq 2 \Gamma(\kappa)$. Using (1.4) yields

$$
\left|\chi_{J^{\prime}(\kappa)}\left(\lambda^{\prime}\right) \operatorname{Im}\left(B\left(\lambda\left(\lambda^{\prime}, \kappa\right), \kappa\right)-\lambda\left(\lambda^{\prime}, \kappa\right)\right)^{-1} \Gamma(\kappa)\right| \leqq c\left|\operatorname{Im}\left(B(\kappa)-\lambda\left(\lambda^{\prime}, \kappa\right)\right)^{-1} \Gamma(\kappa)\right| .
$$

The proof is complete, since

$$
\operatorname{Im}\left(B(\kappa)-\lambda\left(\lambda^{\prime}, \kappa\right)\right)^{-1} \Gamma(\kappa)=\operatorname{Im}\left(-i \Gamma(\kappa)-\lambda^{\prime} \Gamma(\kappa)\right)^{-1} \Gamma(\kappa)=\frac{1}{1+\lambda^{\prime 2}} \in L^{1}(\mathbb{R}) .
$$

Theorem 1.7 (absence of unwanted eigenvalues). If $\mu(\kappa)$ is an eigenvalue of $H(\kappa)$ for $\kappa \in U$, and $\mu(\kappa) \in I$, then $\mu(\kappa)=\lambda(\kappa)$ and $\Gamma(\kappa)=0$. Conversely if $\Gamma(\kappa)=0$ for sufficiently small $\kappa$, then $\lambda(\kappa)$ is an eigenvalue of $H(\kappa)$.

Proof. By assumptions (i) and (iii) of Definition $1.4 \mu(\kappa)$ cannot be an eigenvalue of $\bar{H}(\kappa)$. Hence, for an associated eigenvector $\psi(\kappa), P \psi(\kappa) \neq 0$. Hence

$$
\begin{aligned}
(\mu(\kappa)-z)^{-1} P \psi(\kappa) & =P(H(\kappa)-z)^{-1} \psi(\kappa) \\
& =(B(z, \kappa)-z)^{-1}\left[I+\kappa P W \bar{P}(\bar{H}(\kappa)-z)^{-1} \bar{P}\right] \psi(\kappa)
\end{aligned}
$$

by similar arguments that lead to Theorem 1.2(i). Since by assumption, the term in square brackets applied to $\psi(\kappa)$ remains bounded, $\mu(\kappa)$ must be the unique fixed point of $B(z, \kappa)$. The second statement in the theorem is really just a corollary to Theorem 1.5, for $\Gamma(\kappa) \neq 0$ implies $E_{\kappa}(\{\lambda(\kappa)\}) \stackrel{s}{\rightarrow} P$, hence $E_{\kappa}(\{\lambda(\kappa)\}) \neq 0$ for sufficiently small $\kappa$.

Theorem 1.8 (resonance behaviour). For $\psi \in K,\|\psi\|=1$,

$$
\left|\left\langle\psi, e^{-i H(\kappa) t} \psi\right\rangle\right|=e^{-\Gamma(\kappa) t}+o(1) \text { uniformly in } t .
$$

Proof. By Lemma 1.6

$$
\begin{aligned}
\left\langle\psi, e^{-i H(\kappa) t} \psi\right\rangle & =\left\langle\psi \Lambda e^{-i H(\kappa) t} E_{\kappa}(J(\kappa)) \psi\right\rangle+o(1) \\
& =\pi^{-1} \int_{J(\kappa)} e^{-i \lambda t} \operatorname{Im}(B(\lambda, \kappa)-\lambda)^{-1} d \lambda+o(1) \\
& =\pi^{-1} \int_{\mathbb{R}} e^{-i \lambda t} \operatorname{Im}(B(\kappa)-\lambda)^{-1} d \lambda+o(1) \\
& =e^{-i B(\kappa) t}+o(1)=e^{-i \lambda(\kappa) t} e^{-\Gamma(\kappa) t}+o(1)
\end{aligned}
$$


for $\kappa$ such that $\Gamma(\kappa) \neq 0$, and by Theorem 1.4 and Corollary 1.6

$$
\begin{aligned}
\left\langle\psi, e^{-i H(\kappa) t} \psi\right\rangle & =\left\langle\psi, e^{-i H(\kappa) t} E_{\kappa}(\{\lambda(\kappa)\}) \psi\right\rangle+o(1) \\
& =e^{-i \lambda(\kappa) t}\left\langle\psi, E_{\kappa}(\{\lambda(\kappa)\}) \psi\right\rangle+o(1)=e^{-i \lambda(\kappa) t}+o(1)
\end{aligned}
$$

for $\kappa$ such that $\Gamma(\kappa)=0$.

Remark. In the case $\Gamma_{0}=\operatorname{Im} f\left(\lambda_{0}, 0\right) \neq 0$, we have the interesting result

$$
\left|\left\langle\psi, e^{-i H(\kappa) t} \psi\right\rangle\right|=e^{-\Gamma_{0} \kappa^{2} t}+o(1),
$$

which is known as "Fermi's Golden Rule" in the literature (cf. Reed-Simon [RSIV]). It follows from

$$
\begin{aligned}
\left|e^{-\Gamma(\kappa) t}-e^{-\Gamma_{0} \kappa^{2} t}\right| & \leqq \kappa^{2} t\left|\operatorname{Im} f\left(\lambda_{0}, \kappa\right)-\operatorname{Im} f\left(\lambda_{0}, 0\right)\right| e^{-\kappa^{2} t g(\kappa)}, \\
& \leqq g(\kappa)^{-1}\left|\operatorname{Im} f\left(\lambda_{0}, \kappa\right)-\operatorname{Im} f\left(\lambda_{0}, 0\right)\right| e^{-1} \rightarrow 0,
\end{aligned}
$$

where

$$
g(\kappa):=\inf _{s \in[0, \kappa]}\left|\operatorname{Im} f\left(\lambda_{0}, s\right)\right|
$$

Note particularly, that in this case Theorem 1.7 shows that for $\kappa \neq 0$ there are no eigenvalues of $H(\kappa)$ in the vicinity of $\lambda_{0}$.

We turn to the more general case, where $\lambda_{0}$ is degenerate, of finite multiplicity. We need to tighten assumptions slightly to exclude the occurrence of possible nilpotents in the Livsic-Matrix.

Definition 1.9. We assume Condition 1.3 and define: The operator family $H(\kappa)$ has resonance at $\lambda_{0}$, if there are

- a real neighbourhood $I$ of $\lambda_{0}$,

- a real neighbourhood $U$ of 0 ,

- a densely embedded subspace $\mathscr{H}_{+}$of $\mathscr{H}$ with its dual $\mathscr{H}_{-}$, such that

(i) for $\kappa \in U,(\bar{H}(\kappa)-z)^{-1}$ has a continuous extension from $z \in \mathbb{C} / \mathbb{R}$ onto $z \in I$ as an operator in $B\left(\mathscr{H}_{+} \mathscr{H}_{-}\right)$. This continuation is Lipschitz-continuous with constant $L(\kappa)=o\left(\kappa^{-1}\right)$.

(ii) $K \subset \mathscr{H}_{+}$, and $W(K) \subset \mathscr{H}_{+}$.

(iii) For $\kappa \in U$ and all possible eigenvalues $\mu(\kappa) \in I$ of $H(\kappa)$, the associated eigenvectors are in $\mathscr{H}_{+}$.

(iv) All eigenvalues of $\Lambda$ are simple.

Remark. As in the case of simple resonance, to prove Theorems 1.12 and 1.14, the Lipschitz-continuity in (i) can be replaced by nondegeneracy of $\operatorname{Im} f\left(\lambda_{0}, 0\right)$, cf. [Or] for details.

Lemma 1.10. Let $H(\kappa)$ have resonance at $\lambda_{0}$, then for sufficiently small $\kappa$,

$$
\operatorname{det}(B(\operatorname{Re} z, \kappa)-z)=0
$$

has exactly one solution $z_{j}(\kappa)$ satisfying $\left|z_{j}(\kappa)-\lambda_{0}-\kappa \lambda_{j}\right|=o(\kappa)$. 
Set $\lambda_{j}(\kappa)=\operatorname{Re} z_{j}(\kappa), \Gamma_{j}(\kappa)=-\operatorname{Im} z_{j}(\kappa), B_{j}(\kappa)=B\left(\lambda_{j}(\kappa), \kappa\right)$; choose $\delta_{j}(\kappa)$ such that $\delta_{j}(\kappa)=0$ for $\Gamma_{j}(\kappa)=0$, while for $\Gamma_{j}(\kappa) \neq 0$ and $\kappa \rightarrow 0, \max \left(\frac{\delta_{j}(\kappa)}{\kappa}, \frac{\kappa^{\gamma} \delta_{j}(\kappa)}{\Gamma_{j}(\kappa)}\right.$, $\left.\frac{\kappa^{2} L(\kappa) \delta_{j}(\kappa)}{\Gamma_{j}(\kappa)}\right) \rightarrow 0$ for some $\gamma<2$, but at the same time $\frac{\Gamma_{j}(\kappa)}{\delta_{j}(\kappa)} \rightarrow 0$; finally set $J_{j}(\kappa)=\left[\lambda_{j}(\kappa)-\delta_{j}(\kappa), \lambda_{j}(\kappa)+\delta_{j}(\kappa)\right]$. Then for $\lambda \in J_{j}(\kappa)$,

$$
\left|\left(B_{j}(\kappa)-\lambda\right)^{-1}\right| \leqq c / \Gamma_{j}(\kappa),
$$

and $P_{j}(\kappa)$, the projection onto the $z_{j}(\kappa)$-associated eigenvector of $B_{j}(\kappa)$ satisfies

$$
\left|\left(B_{j}(\kappa)-\lambda\right)^{-1}\left(I-P_{j}(\kappa)\right)\right| \leqq c / \kappa
$$

for $\lambda \in J_{j}(\kappa)$.

Proof. Substituting $z=\lambda_{0}+\kappa y$ for $\kappa \neq 0$ makes (1.9) equivalent to

$$
\operatorname{det}\left(\Lambda-\kappa f\left(\lambda_{0}+\kappa \operatorname{Re} y, \kappa\right)-y\right)=0 .
$$

The iteration (over $m$ ): "Solve

$$
\operatorname{det}\left(\Lambda-\kappa f\left(\lambda_{0}+\kappa \operatorname{Re} y_{j}^{(m-1)}(\kappa), \kappa\right)-y\right)=0
$$

and choose $y_{j}^{(m)}(\kappa)$ as the continuous solution satisfying $y_{j}^{(m)}(0)=\lambda_{j}$ " converges to a solution $y_{j}(\kappa)$, satisfying $y_{j}(0)=\lambda_{j}$. Set $z_{j}(\kappa)=\lambda_{0}+\kappa y_{j}(\kappa)$. The estimates (1.10) and (1.11) are easily derived by using Cramer's formula on $\Lambda-\kappa f\left(\lambda_{j}(\kappa), \kappa\right)$.

The analogon to Lemma 1.6 is

Lemma 1.11. Let $H(\kappa)$ have resonance at $\lambda_{0}$. Using the notation in Lemma 1.10, we have

$$
\chi_{J_{j}(\kappa)}(\lambda) \operatorname{Im}(B(\lambda, \kappa)-\kappa)^{-1}-P_{j}(\kappa)^{*} \operatorname{Im}\left(B_{j}(\kappa)-\lambda\right)^{-1} P_{j}(\kappa) \rightarrow 0
$$

in the $L^{1}(\mathbb{R})$-norm with respect to $\lambda$ as $\kappa \rightarrow 0$.

Proof. Again to control the simultaneous divergence of the two terms, we substitute $\lambda^{\prime}=\Gamma_{j}(\kappa)^{-1}\left(\lambda-\lambda_{j}(\kappa)\right)$. Proceeding as in Lemma 1.6 yields pointwise convergence of

$$
\begin{gathered}
\chi_{J^{\prime}(\kappa)}\left(\lambda^{\prime}\right) \operatorname{Im}\left(B\left(\lambda_{j}\left(\lambda^{\prime}, \kappa\right), \kappa\right)-\lambda_{j}\left(\lambda^{\prime}, \kappa\right)\right)^{-1} \Gamma_{j}(\kappa) \\
-\chi_{J_{j}^{\prime}(\kappa)}\left(\lambda^{\prime}\right) \operatorname{Im}\left(B_{j}(\kappa)-\lambda_{j}\left(\lambda^{\prime}, \kappa\right)\right)^{-1} \Gamma_{j}(\kappa) \rightarrow 0 .
\end{gathered}
$$

Now notice that, remembering $J_{j}^{\prime}(\kappa)=\left[-\frac{\delta_{j}(\kappa)}{\Gamma_{j}(\kappa)}, \frac{\delta_{j}(\kappa)}{\Gamma_{j}(\kappa)}\right]$,

$$
\begin{aligned}
& \left|\chi_{J_{j}^{\prime}(\kappa)}\left(\lambda^{\prime}\right) \operatorname{Im}\left(B\left(\lambda_{j}\left(\lambda^{\prime}, \kappa\right), \kappa\right)-\lambda_{j}\left(\lambda^{\prime}, \kappa\right)\right)^{-1} \Gamma_{j}(\kappa)\right| \\
& \leqq\left|\chi_{J_{j}^{\prime}(\kappa)}\left(\lambda^{\prime}\right)\left(B\left(\lambda_{j}\left(\lambda^{\prime}, \kappa\right), \kappa\right)^{*}-\lambda_{j}\left(\lambda^{\prime}, \kappa\right)\right)^{-1} \operatorname{Im} B_{j}(\kappa)\left(B\left(\lambda_{j}\left(\lambda^{\prime}, \kappa\right), \kappa\right)-\lambda_{j}\left(\lambda^{\prime}, \kappa\right)\right)^{-1} \Gamma_{j}(\kappa)\right| \\
& \quad+\left|\chi_{J^{\prime}(\kappa)}\left(\lambda^{\prime}\right)\left(B\left(\lambda_{j}\left(\lambda^{\prime}, \kappa\right), \kappa\right)^{*}-\lambda_{j}\left(\lambda^{\prime}, \kappa\right)\right)^{-1} \kappa^{2} L(\kappa) \delta_{j}(\kappa)\left(B\left(\lambda_{j}\left(\lambda^{\prime}, \kappa\right), \kappa\right)-\lambda_{j}\left(\lambda^{\prime}, \kappa\right)\right)^{-1} \Gamma_{j}(\kappa)\right| \\
& \leqq 4\left|\chi_{J_{j}^{\prime}(\kappa)}\left(\lambda^{\prime}\right) \operatorname{Im}\left(B_{j}(\kappa)-\lambda_{j}\left(\lambda^{\prime}, \kappa\right)\right)^{-1} \Gamma_{j}(\kappa)\right|+4\left|\chi_{J_{j}^{\prime}(\kappa)}\left(\lambda^{\prime}\right)\left(B_{j}(\kappa)-\lambda_{j}\left(\lambda^{\prime}, \kappa\right)\right)^{-1} \Gamma_{j}(\kappa)\right|^{2},
\end{aligned}
$$


and

$$
\begin{aligned}
\chi_{J_{j}^{\prime}(\kappa)}\left(\lambda^{\prime}\right) \operatorname{Im}\left(B_{j}(\kappa)-\lambda_{j}\left(\lambda^{\prime}, \kappa\right)\right)^{-1} \Gamma_{j}(\kappa) \\
=-\chi_{J_{j}^{\prime}(\kappa)}\left(\lambda^{\prime}\right)\left(B_{j}(\kappa)^{*}-\lambda_{j}\left(\lambda^{\prime}, \kappa\right)\right)^{-1} \operatorname{Im} B_{j}(\kappa)\left(B_{j}(\kappa)-\lambda_{j}\left(\lambda^{\prime}, \kappa\right)\right)^{-1} \Gamma_{j}(\kappa) \\
=\chi_{J^{\prime}(\kappa)}\left(\lambda^{\prime}\right) P_{j}(\kappa)^{*} \operatorname{Im}\left(B_{j}(\kappa)-\lambda_{j}\left(\lambda^{\prime}, \kappa\right)\right)^{-1} P_{j}(\kappa) \Gamma_{j}(\kappa) \\
\quad-\chi_{J_{j}^{\prime}(\kappa)}\left(\lambda^{\prime}\right)\left[\left(B_{j}(\kappa)-\lambda_{j}\left(\lambda^{\prime}, \kappa\right)\right)^{-1} P_{j}(\kappa) \Gamma_{j}(\kappa)\right]^{*} \operatorname{Im} B_{j}(\kappa)\left(B_{j}(\kappa)-\lambda_{j}\left(\lambda^{\prime}, \kappa\right)\right)^{-1}\left(I-P_{j}(\kappa)\right) \\
\quad-\chi_{J_{j}^{\prime}(\kappa)}\left(\lambda^{\prime}\right)\left[\operatorname{Im} B_{j}(\kappa)\left(B_{j}(\kappa)-\lambda_{j}\left(\lambda^{\prime}, \kappa\right)^{-1}\left(I-P_{j}(\kappa)\right)\right]^{*}\left(B_{j}(\kappa)-\lambda_{j}\left(\lambda^{\prime}, \kappa\right)\right)^{-1} \Gamma_{j}(\kappa) .\right.
\end{aligned}
$$

Using (1.10), (1.11) and $\left|\operatorname{Im} B_{j}(\kappa)\right| \leqq c \kappa^{2}$, we bound terms two and three in (1.15) by $c \kappa$. Hence we have established pointwise convergence for the substituted (1.12).

We turn to finding an $L^{1}$-dominant. By assumption $\delta_{j}(\kappa) / \Gamma_{j}(\kappa) \leqq c \kappa^{-\gamma}$ for some $\gamma<2$, hence $\kappa \chi_{J_{j}^{\prime}(\kappa)}\left(\lambda^{\prime}\right) \leqq\left|\lambda^{\prime}\right|^{-1 / \gamma}$. Using this,

and

$$
P_{j}(\kappa)^{*} \operatorname{Im}\left(B_{j}(\kappa)-\lambda_{j}\left(\lambda^{\prime}, \kappa\right)\right)^{-1} P_{j}(\kappa)=P_{j}(\kappa)^{*} \frac{1}{1+\lambda^{\prime 2}} P_{j}(\kappa)
$$

$$
\begin{aligned}
& \left(B_{j}(\kappa)-\lambda_{j}\left(\lambda^{\prime}, \kappa\right)\right)^{-1} \Gamma_{j}(\kappa) \\
& \quad=\left(z_{j}(\kappa)-\lambda_{j}\left(\lambda^{\prime}, \kappa\right)\right)^{-1} \Gamma_{j}(\kappa) P_{j}(\kappa)+\left(B_{j}(\kappa)-\lambda_{j}\left(\lambda^{\prime}, \kappa\right)\right)^{-1}\left(I-P_{j}(\kappa)\right) \Gamma_{j}(\kappa) \\
& \quad=\left(-i-\lambda^{\prime}\right)^{-1} P_{j}(\kappa)+\left(B_{j}(\kappa)-\lambda_{j}\left(\lambda^{\prime}, \kappa\right)\right)^{-1}\left(I-P_{j}(\kappa)\right) \Gamma_{j}(\kappa),
\end{aligned}
$$

as well as the fact that

$$
\left|\chi_{J_{j}^{\prime}(\kappa)}\left(\lambda^{\prime}\right)\left(B_{j}(\kappa)-\lambda_{j}\left(\lambda^{\prime}, \kappa\right)\right)^{-1} \Gamma_{j}(\kappa)\right| \leqq c\left(\frac{1}{1+\left|\lambda^{\prime}\right|}+\frac{1}{1+\left|\lambda^{\prime}\right|^{1 / \gamma}}\right) \in L^{2}\left(d \lambda^{\prime}\right),
$$

yields $L^{1}$-dominants for all terms on the right-hand side of (1.15) and the remaining second term of (1.14). The lemma is proven, since

$$
\chi_{\mathbb{R} \backslash J_{j}^{\prime}(\kappa)}\left(\lambda^{\prime}\right) P_{j}(\kappa)^{*} \frac{1}{1+\lambda^{\prime 2}} P_{j}(\kappa) \rightarrow 0 \quad \text { in } \quad L^{1}\left(d \lambda^{\prime}\right) .
$$

Theorem 1.12 (spectral concentration). Let $H(\kappa)$ have resonance at $\lambda_{0}$. With the notations in Lemma 1.10,

$$
E_{\kappa}\left(J_{j}(\kappa)\right) \rightarrow P_{j} \quad(\kappa \rightarrow 0),
$$

where $P_{j}=P_{j}(0)$ is the orthogonal projection onto the $\lambda_{j}$-associated eigenspace of $\Lambda$.

Theorem 1.13 (absence of unwanted eigenvalues). If $\mu(\kappa)$ is an eigenvalue of $H(\kappa)$ for $\kappa \in U$, and $\mu(\kappa) \in I$, then $\mu(\kappa)=\lambda_{j}(\kappa)$ for one of the $j=1, \ldots, n$, and $\Gamma_{j}(\kappa)=0$.

Conversely if one of the $\Gamma_{j}(\kappa)=0$ for sufficiently small $\kappa$, then the corresponding $\lambda_{j}(\kappa)$ is an eigenvalue of $H(\kappa)$.

Theorem 1.14 (resonance behaviour). For $\psi \in K$,

$$
\left\langle\psi, e^{-i H(\kappa) t} \psi\right\rangle=\sum_{j=1}^{n} a_{j} e^{-i \lambda_{j}(\kappa) t} e^{-\Gamma_{j}(\kappa) t}+o(1)
$$

uniformly in $t$, where $a_{j}=\left\|P_{j} \psi\right\|^{2}$.

We omit the proofs of these theorems and turn to applications. 


\section{Resonances and Analytic Perturbation Theory}

In this section we shall compare the theory developed in Sect. 1 with methods of treating resonances using analytic perturbation theory.

Theorem 2.1 (eigenvalues). Let $H_{0}$ be a self-adjoint operator in the Hilbertspace $\mathscr{H}$, $\lambda_{0}$ an isolated eigenvalue of $H_{0}$ of finite multiplicity. Let $W$ be symmetric and either relatively bounded or relatively form-bounded with respect to $H_{0}$. If $W$ satisfies the nondegeneracy assumption in Definition 1.9 (iv), then $H(\kappa):=H_{0}+\kappa W$ has resonance at $\lambda_{0}$ in the sense of Definition 1.9.

In fact solutions $z_{j}(\kappa)$ of

$$
\operatorname{det}\left((B(\operatorname{Re} z, \kappa)-z)^{-1}\right)=0
$$

satisfying $z_{j}(\kappa)=\lambda_{0}+\kappa \lambda_{j}+o(\kappa)$ are real and analytic and represent all eigenvalues of $H(\kappa)$ near $\lambda_{0}$.

Proof. The proof is a straightforward application of Theorem 1.13. Note that $(\bar{H}(\kappa)-z)^{-1}$ is analytic in both $\kappa$ and $z$ near 0 and $\lambda_{0}$, in particular $\operatorname{Im} B(\lambda, \kappa)=0$ for small $\kappa$, and in Definition $1.9 \mathscr{H}_{+}$can be chosen to be $\mathscr{H}$.

Theorem 2.2 (resonances). Assume Conditions 1.3 to hold, in addition assume that Assumptions 1.9 hold, except 1.9(i), in fact, replacing 1.9(i) we assume that for $\kappa \in U$ $(\bar{H}(\kappa)-z)^{-1}$ can be analytically continued across the real axis onto a complex neighbourhood of $\lambda_{0}$, as an operator from $\mathscr{H}_{+}$to $\mathscr{H}_{-}$. Then for sufficiently small $\kappa$,

$$
\operatorname{det}(B(z, \kappa)-z)=0
$$

has exactly one solution $\hat{z}_{j}(\kappa)$ satisfying $\hat{z}_{j}(\kappa)=\lambda_{0}+\kappa \lambda_{j}+o(\kappa)$.

As in Lemma 1.10, set $\hat{\lambda}_{j}(\kappa)=\operatorname{Re} \hat{z}_{j}(\kappa), \hat{\Gamma}_{j}(\kappa)=-\operatorname{Im} \hat{z}_{j}(\kappa) ;$ choose $\hat{\delta}_{j}(\kappa)$ such that $\hat{\delta}_{j}(\kappa)=0$ for $\hat{\Gamma}_{j}(\kappa)=0$, while for $\hat{\Gamma}_{j}(\kappa) \neq 0$ and $\kappa \rightarrow 0, \max \left(\hat{\delta}_{j}(\kappa) / \kappa, \kappa^{\nu} \hat{\delta}_{j}(\kappa) / \hat{\Gamma}_{j}(\kappa)\right) \rightarrow 0$ for some $\gamma<2$, but $\hat{\Gamma}_{j}(\kappa) / \hat{\delta}_{j}(\kappa) \rightarrow 0$; set $\hat{J}_{j}(\kappa)=\left[\hat{\lambda}_{j}(\kappa)-\hat{\delta}_{j}(\kappa), \hat{\lambda}_{j}(\kappa)+\hat{\delta}_{j}(\kappa)\right]$, and let $\hat{P}_{j}(\kappa)$ be the projection onto the $\hat{z}_{j}(\kappa)$-associated eigenvector of $B\left(\hat{z}_{j}(\kappa), \kappa\right)$. Then

(i) $E_{\kappa}\left(\hat{J}_{j}(\kappa)\right) \stackrel{s}{\rightarrow} P_{j}$,

(ii) there are no eigenvalues of $H(\kappa)$ near $\lambda_{0}$ that are not solutions of (2.1),

(iii) $\left\langle\psi, e^{-i H(\kappa) t} \psi\right\rangle=\sum_{j=1}^{n} a_{j} e^{-i \hat{\lambda}_{j}(\kappa) t} e^{-\hat{\Gamma}_{j}(\kappa) t}+o(1)$, for $\psi \in K$ and $a_{j}=\left\|P_{j} \psi\right\|^{2}$.

Proof. Assumption 1.9(i) is actually implied by the stronger analyticity requirement, in fact $L(\kappa)$ is then constant. Hence Theorems 1.12, 1.13, and 1.14 hold for solutions $z_{j}(\kappa)$ of Eq. (1.9). However, these are not a priori solutions of (2.1). Solutions of (2.1) can be found using the corresponding iteration as in the proof of Lemma 1.10. Now, Lipschitz-continuity yields

$$
\left|f\left(\hat{z}_{j}(\kappa), \kappa\right)-f\left(\lambda_{j}(\kappa), \kappa\right)\right| \leqq L\left(\left|\hat{\lambda}_{j}(\kappa)-\lambda_{j}(\kappa)\right|+\hat{\Gamma}_{j}(\kappa)\right),
$$

and the nondegeneracy of the eigenvalues of $\Lambda$ lets us transfer this estimate to

hence

$$
\left|\hat{y}_{j}(\kappa)-y_{j}(\kappa)\right| \leqq c_{1} \kappa\left(\left|\hat{\lambda}_{j}(\kappa)-\lambda_{j}(\kappa)\right|+\hat{\Gamma}_{j}(\kappa)\right),
$$

$$
\left|\hat{z}_{j}(\kappa)-z_{j}(\kappa)\right| \leqq c_{1} \kappa^{2}\left(\left|\hat{\lambda}_{j}(\kappa)-\lambda_{j}(\kappa)\right|+\hat{\Gamma}_{j}(\kappa)\right),
$$


and in particular

$$
\left|\hat{\lambda}_{j}(\kappa)-\lambda_{j}(\kappa)\right| \leqq c_{2} \kappa^{2} \hat{\Gamma}_{j}(\kappa) \text { and }\left|\hat{\Gamma}_{j}(\kappa)-\Gamma_{j}(\kappa)\right| \leqq c_{2} \kappa^{2} \hat{\Gamma}_{j}(\kappa)
$$

Having chosen $\hat{J}_{j}(\kappa)$ as in the theorem, note that $\lambda_{j}(\kappa) \in \hat{J}_{j}(\kappa)$ and $\delta_{j}(\kappa)$ can be chosen to satisfy conditions in Lemma 1.10 , and $\hat{J}_{j}(\kappa)=J_{j}(\kappa)$. This proves (i). (ii) follows from Theorem 1.13 and $\hat{\Gamma}_{j}(\kappa)=0 \Leftrightarrow \Gamma_{j}(\kappa)=0$. (iii) is a consequence of Theorem 1.14 and

$$
\begin{aligned}
\left|e^{-i \hat{z}_{j}(\kappa) t}-e^{-i z_{j}(\kappa) t}\right| & \leqq c \kappa^{2} t \min \left\{\hat{\Gamma}_{j}(\kappa), \Gamma_{j}(\kappa)\right\} \max \left\{e^{-\hat{\Gamma}_{j}(\kappa) t}, e^{-\Gamma_{j}(\kappa) t}\right\} \\
& \leqq c \kappa^{2} e^{-1} \rightarrow 0 \quad \text { for } \kappa \rightarrow 0 .
\end{aligned}
$$

Remark. (i) Howland has proven a similar result in [Ho]. He uses analyticity of $B(z, \kappa)$, not of $(\bar{H}(\kappa)-z)^{-1}$, he needs no non-degeneracy conditions on $\Lambda$. Using function theoretic arguments, as he does, nilpotents can be treated. He cannot prove absence of unwanted eigenvalues as in (ii), since he makes no assumptions on $(\bar{H}(\kappa)-z)^{-1}$ outside of $K$.

(ii) Simon [S1] treats the many body Schrödinger operator $H(\kappa)=-\Delta+V+\kappa W$, which we shall turn to in the next section, for dilation-analytic potentials $V$ and $W$. Using the technique of Aguilar-Combes [AC], Balslev-Combes [BC], he continues the resolvent $\left\langle\psi,(H(\kappa)-z)^{-1} \psi\right\rangle$ for $\psi$ in the densely embedded subspace of dilation-analytic vectors. Then using analytic perturbation theory of Kato and Rellich [Ka] applied to the non-self-adjoint dilated operator $H(\kappa, \Theta)$ he identifies complex poles in the second sheet of the continued resolvent. He calls them resonances. We shall show at the end of the next section, in Corollary 3.7, how the assumptions of Theorem 2.2 are satisfied in his case.

(iii) Theorem 2.2 underlines the asymptotic character of our definition of resonance. Resonance width as considered here is not a number nor a function of $\kappa$, but in actual fact an equivalence class of functions, where the equivalence relation is given by (we just consider the case of simple resonance)

$$
\Gamma(\kappa) \sim \tilde{\Gamma}(\kappa) \Leftrightarrow \frac{\Gamma(\kappa)}{\hat{\Gamma}(\kappa)} \rightarrow 1 .
$$

For if $\Gamma(\kappa) \sim \hat{\Gamma}(\kappa)$, then the corresponding exponents in the time decay estimate of Theorems 1.8 and 1.14 can just be interchanged, the difference just disappears in the $o(1)$ term in the decay estimate. Theorem 2.2 then just states: the resonance widths in the analytic sense and in our sense are equivalent.

(iv) Nonetheless, the question may be asked, if perhaps $\Gamma(\kappa)$ and $\hat{\Gamma}(\kappa)$ respectively $\lambda(\kappa)$ and $\hat{\lambda}(\kappa)$ lie even closer together. For many body Schrödinger Operators this question is not particularly interesting, since by a Paley-Wiener argument the $o(1)$ term must occur, cf. [S2, Her] and others. As Herbst pointed out in [Her], for operators with the whole axis contained in their spectrum, the $o(1)$ term is not compulsory, in fact, for the Stark Operator, which is easily made to fit our definition of resonance (cf. [OP] for details) be proved that this term can be dispensed with.

Generically, however, (2.2) should be optimal, because

$$
\hat{\Gamma}(\kappa) \leqq|\hat{z}(\kappa)-\lambda(\kappa)|
$$


should imply

$$
\hat{\Gamma}(\kappa) \leqq c_{3}|\operatorname{Im} f(\hat{z}(\kappa), \kappa)-\operatorname{Im} f(\lambda(\kappa), \kappa)|,
$$

if $f$ is a nondegenerate analytic function. Hence we would have

$$
\hat{\Gamma}(\kappa) \leqq c_{3} \frac{1}{\kappa^{2}}|\hat{\Gamma}(\kappa)-\Gamma(\kappa)|
$$

It may be possible, by making $K$ the space with respect to which the Livsic-Matrix was formed depend suitably upon $\kappa$, to improve on the term $\kappa^{2}$, which prefixes $f(z, \kappa)$ in the expression for the Livsic Matrix, and to thus reduce the difference between $\hat{\Gamma}(\kappa)$ and $\Gamma(\kappa)$.

\section{The Many Body Problem}

As the principal application of the notions introduced in the first section, we shall prove existence of resonances in the quantum-mechanical many body problem for a very general class of potentials. We emphasize that we require only differentiability and decay properties and no restrictions regarding analyticity and symmetry of the potentials.

The resonances that occur are perturbations of embedded eigenvalues of approximating systems, in which certain interaction-potentials are assumed to vanish. For the Helium atom (i.e. the three body problem) this is known as the Auger-effect [RSIV] (in the approximating system the $e$-e-interaction is neglected).

Our method of proof consists in firstly establishing a uniform Mourré-type positivity estimate for the commutator $[\bar{H}(\kappa), A]$, where $A$ is the generator of dilations: Corollary 3.2. Equipped with this estimate we shall use Mourrés original theorem on absence of singular continuous spectrum [Mo], and an extension due to Jensen, Mourré and Perry [JMP] in order to establish validity of assumptions in Definition 1.4.

Let $X=\mathbb{R}^{m}, \mathscr{H}=L^{2}(X), X_{j} \subset X$ be subspaces, $\mathscr{H}\left(X_{j}\right)=L^{2}\left(X_{j}\right), \pi_{j}$ be orthogonal projections satisfying $R\left(\pi_{j}\right)=X_{j}$, let $V_{j}$ be interaction potentials such that $V_{j} \in \mathscr{S}^{\prime}\left(X_{j}\right) \cap L_{\text {loc }}^{1}\left(X_{j}\right)$. Furthermore let $\mathscr{H}_{2}, \mathscr{H}_{2}\left(X_{j}\right)$ be $D\left(\Delta_{X}\right), D\left(\Delta_{X_{j}}\right)$ respectively, and $\mathscr{H}_{-2}, \mathscr{H}_{-2}\left(X_{j}\right)$ the corresponding duals.

$$
H:=-\Delta_{X}+\Sigma V_{j}\left(\pi_{j}(x)\right)
$$

is the generalized many body Schrödinger operator that we shall consider here. Denote by $\mathscr{T}(H)$ the set of thresholds of $H$, i.e. the eigenvalues of all subsystems of $H$, and let

$$
A=\frac{1}{2}\left(x \cdot \nabla_{X}+\nabla_{X} \cdot x\right)
$$

be the skew-adjoint generator of the dilatation group in $\mathscr{H}$.

For these operators Perry, Sigal, and Simon [PSS], and later Froese, Herbst [FH1] have proven the following theorem:

Theorem 3.1 (Perry, Sigal, Simon). Let $X, X_{j}, V_{j}$, and $H$ be as above, $\lambda_{0} \in \mathbb{R} \backslash \mathscr{T}(H)$. Let the $V_{j}$ satisfy the assumptions

(i) $V_{j} \in B\left(\mathscr{H}_{2}\left(X_{j}\right), \mathscr{H}\left(X_{j}\right)\right)$ and compact

(ii) $x_{X_{j}} \cdot \nabla_{X_{j}} V_{j} \in B\left(\mathscr{H}_{2}\left(X_{j}\right), \mathscr{H}_{-2}\left(X_{j}\right)\right)$ and compact. 
Then there is an open interval $I, \lambda_{0} \in I$, an $\alpha>0$ and a compact operator $K$, so that

$$
E_{H}(I)[H, A] E_{H}(I) \geqq \alpha E_{H}(I)+K .
$$

Furthermore, the set of thresholds $\mathscr{T}(H)$ is countable and eigenvalues of $H$ accumulate only in points of $\mathscr{T}(H)$.

We are here interested in the following perturbed version of this theorem (a similar result appears in [AHS] by Agmon, Herbst, and Skibsted):

Corollary 3.2. Let $H$ be as in Theorem 3.1, in addition let $W \in \mathscr{S}^{\prime}(X) \cap L_{\text {loc }}^{1}(X)$ satisfy

(i) $W \in B\left(\mathscr{H}_{2}, \mathscr{H}\right)$

(ii) $x \cdot \nabla_{X} W \in B\left(\mathscr{H}_{2}, \mathscr{H}_{-2}\right)$,

and define

$$
H(\kappa):=H+\kappa W \quad \text { on } \quad D(H(\kappa))=\mathscr{H}_{2} .
$$

Let $\lambda_{0}$ be an eigenvalue but no threshold of $H$. Let $P$ be the corresponding (finitedimensional) eigenprojection, $\bar{P}=I-P, \bar{H}(\kappa)=\bar{P} H(\kappa) \bar{P}$. Then for an appropriate zero-neighbourhood $U$ and an interval I around $\lambda_{0}$ there is an $\alpha \geqq 0$, such that for $\kappa \in U$

$$
\bar{E}_{\kappa}(I)[\bar{H}(\kappa), A] \bar{E}_{\kappa}(I) \geqq \alpha \bar{E}_{\kappa}(I) .
$$

Here $\bar{E}_{\kappa}$ is the spectral resolution for $\bar{H}(\kappa)$.

Proof. Notice that

$$
\bar{E}_{0}(I)[\bar{H}(0), A] \bar{E}_{0}(I) \geqq \alpha \bar{E}_{0}(I)+\bar{K}
$$

follows directly from (3.2), where $\bar{K}=\bar{P} K \bar{P}$, and hence for appropriate $I^{\prime} \subset I$ and $\alpha^{\prime} \in(0, \alpha)$,

$$
\bar{E}_{0}\left(I^{\prime}\right)[\bar{H}(0), A] \bar{E}_{0}\left(I^{\prime}\right) \geqq \alpha^{\prime} \bar{E}_{0}\left(I^{\prime}\right) .
$$

Now, the range of the projector $P$ lies in $\mathscr{H}_{2} \cap D(A)$, hence conditions (i) and (ii) for $W$ carry over to $\bar{W}=\bar{P} W \bar{P}$. Therefore $[H(\kappa), A] \in B\left(\mathscr{H}_{2}, \mathscr{H}_{-2}\right)$ and normcontinuous in $\kappa$. For $f \in C_{0}^{\infty}\left(I^{\prime}\right), f(H(\kappa)) \in B\left(\mathscr{H}, \mathscr{H}_{2}\right)$ and continuous, so that by choosing $f=1$ on some $I^{\prime \prime} \subset I$ and an appropriate $\alpha^{\prime \prime} \in\left(0, \alpha^{\prime}\right),(3.4)$ implies (3.2), just set $\alpha=\alpha^{\prime \prime}, I=I^{\prime \prime}$.

Theorem 3.3 (Mourré). Let $H(\kappa), \lambda_{0}, P, \bar{H}(\kappa)$ be as above, $\mathscr{H}_{2}, \mathscr{H}_{-2}$ as introduced before Theorem 3.1, let the $V_{j}$ satisfy

(iii) $x_{X_{j}} \cdot \nabla_{X_{j}} V_{j} \in B\left(\mathscr{H}_{2}\left(X_{j}\right), \mathscr{H}\left(X_{j}\right)\right)$

(iv) $\left(x_{X_{j}} \cdot \nabla_{X_{j}}\right)^{2} V_{j} \in B\left(\mathscr{H}_{2}\left(X_{j}\right), \mathscr{H}_{-2}\left(X_{j}\right)\right)$,

in addition to (i) and (ii) in Theorem 3.1. Let $A$ be the skew-adjoint generator of dilatations. Then

(a) $D(A) \cap \mathscr{H}_{2}$ is dense in $\mathscr{H}_{2}$.

(b) $T(t)=e^{t A}$ leaves $\mathscr{H}_{2}$ invariant for $\psi \in \mathscr{H}_{2}: \sup _{|t| \leqq 1}\|T(t) \psi\|_{2}<\infty$.

(c) $B_{1}(\kappa)=[\bar{H}(\kappa), A] \in B\left(\mathscr{H}_{2}, \mathscr{H}\right)$ with a locally uniformly bounded norm.

(d) $B_{2}(\kappa)=\left[B_{1}(\kappa), A\right] \in B\left(\mathscr{H}_{2}, \mathscr{H}_{-2}\right)$ again with a locally uniformly bounded norm. 
(e) There is an open zero-neighbourhood $U$, a compact interval I, and an $\alpha>0$, such that for all $\kappa \in U$ :

$$
\bar{E}_{\kappa}(I) B_{1}(\kappa) \bar{E}_{\kappa}(I) \geqq \alpha \bar{E}_{\kappa}(I),
$$

where $\bar{E}_{\kappa}$ is the spectral resolution for $\bar{H}(\kappa)$.

Furthermore for any compact interval $I^{\prime} \subset I^{\text {, }}$

$$
\lim _{\mu_{\searrow} 0}(|A|+1)^{-1}(\bar{H}(\kappa)-\lambda-i \mu)^{-1}(|A|+1)^{-1}
$$

exists and is uniformly norm-continuous in $I^{\prime} \times U$.

Remark. The actual content of Mourré's theorem is proving (3.5), assuming (a)-(e) in an abstract setting. Nowadays this proof is well known and shall be omitted. In particular $\kappa$-dependence causes no new problems. Establishing (a)-(d) for the case considered here is delicate, but the techniques are well known. Details can be found in [Or]. Again $\kappa$-dependence demands nothing new. Lastly, (e) has just been established in Corollary 3.2.

Theorem 3.4 (Jensen, Mourré, Perry). Let $H(\kappa), \lambda_{0}, P, \bar{H}(\kappa), \mathscr{H}_{2}, \mathscr{H}_{-2}$ be as above, in addition to (i), (ii) in Theorem 3.1 and (iii), (iv) in Theorem 3.3, let the $V_{j}$ satisfy

(v) $\left(x_{X_{j}} \cdot \nabla_{X_{j}}\right)^{2} V_{j} \in B\left(\mathscr{H}_{2}\left(X_{j}\right), \mathscr{H}\left(X_{j}\right)\right)$,

(vi) $\left(x_{X_{j}} \cdot \nabla_{X_{j}}\right)^{3} V_{j} \in B\left(\mathscr{H}_{2}\left(X_{j}\right), \mathscr{H}_{-2}\left(X_{j}\right)\right)$.

Then in addition to (a)-(e) in Theorem 3.3,

(c') $B_{2}(\kappa) \in B\left(\mathscr{H}_{2}, \mathscr{H}\right)$ with a locally uniformly bounded norm,

(d') $B_{3}(\kappa):=\left[B_{2}(\kappa), A\right] \in B\left(\mathscr{H}_{2}, \mathscr{H}_{-2}\right)$ with a locally uniformly bounded norm. Furthermore for any $I^{\prime} \subset \stackrel{I}{ }$,

$$
\lim _{\mu_{\searrow} 0}(|A|+1)^{-2}(\bar{H}(\kappa)-\lambda-i \mu)^{-2}(|A|+1)^{-2}
$$

exists and is uniformly bounded in $I^{\prime} \times U$.

Remark. Again the actual content of the theorem is proving (3.6) from (a)-(e), (c'), $\left(d^{\prime}\right)$. In fact in their paper [JMP] Jensen, Mourre and Perry have established the link between controlling higher orders of commutators and continuity of higher powers of resolvents.

We are now in position to prove existence of resonances for $H(\kappa)$.

Theorem 3.5. Let $H(\kappa), \lambda_{0}, P, \bar{H}(\kappa)$ be as in Corollary 3.2, let the $V_{j}$ satisfy the assumptions of Theorem 3.4. In addition, let $W$ satisfy

$$
\left(x \cdot \nabla_{X}\right)^{2} W \in B\left(\mathscr{H}_{2}, \mathscr{H}\right),
$$

and let all eigenvalues of $P W P$ be simple. Then $H(\kappa)$ fulfills requirements of Definitions 1.4 or 1.9. Hence Theorems $1.5,1.7$ and 1.8 or respectively 1.13,1.14, and 1.15 apply.

Proof. We choose $I$ and $U$ as in Theorem 3.4, and $\mathscr{H}_{+}=D\left(A^{2}\right)$. By Theorem 3.3 $(\bar{H}(\kappa)-z)^{-1}$ has the required continuous extension, and by Theorem 3.4 it is Lipschitz-continuous, even with a uniformly bounded constant. Hence (i) holds in Definition 1.4 and 1.9. (ii) and (iii) are consequences of decay and regularity properties of eigenfunctions of the operators considered: Froese and Herbst 
[FH2] have proven (more than) the necessary decay estimates, conditions on the $V_{j}$ imply that eigenfunctions are in $D\left(A^{2}\right)$, and the conditions on $W$ imply that $W$ leaves $D\left(A^{2}\right)$ invariant (details for (ii) and (iii) can be found in [Or] and [AHS]).

As a promised at the end of section two, we conclude by making the connection to the analytic case that was treated by Simon in [S1]. To do this, we first state the analogon of Theorem 3.1 for dilation analytic interaction potentials. The theorem was proved in $[\mathrm{BC}]$ by Balslev, Combes.

Theorem 3.6 (Balslev, Combes). Let $X, X_{j}, V_{j}, H$ be as before Theorem 3.1, let the $V_{j}$ satisfy the assumptions:

(i) $V_{j} \in B\left(\mathscr{H}_{2}\left(X_{j}\right), \mathscr{H}\left(X_{j}\right)\right)$ and compact,

(ii) $V_{j}(\Theta):=e^{\Theta A} V_{j} e^{-\Theta A}$ has an analytic continuation onto the strip $|\operatorname{Im} \Theta|<\alpha$, for some $\alpha>0$.

Let $\mathscr{H}_{\beta}:=R\left(e^{-\beta|A|}\right)$, for $\beta<\alpha$, a set of dilation analytic vectors, then

(i) the point spectrum of $H$ is bounded and the set of its accumulation points is contained in $\mathscr{T}(H)$,

(ii) the eigenspace corresponding to an eigenvalue $\lambda_{0} \in \mathbb{R} \backslash \mathscr{T}(H)$ is in $\mathscr{H}_{\beta}$,

(iii) the essential spectrum of $H(\Theta):=e^{\Theta A} H e^{-\Theta A}$ is $\mathscr{T}(H)+e^{-2 \Theta} \mathbb{R}^{+}$,

(iv) eigenvalues of $H$, not in $\mathscr{T}(H)$, are eigenvalues of $H(\Theta)$.

Corollary 3.7. Let $H$ be as in Theorem 3.6, $W \in \mathscr{S}^{\prime}(X) \cap L_{\text {loc }}^{1}(X)$ satisfy

(i) $W \in B\left(\mathscr{H}_{2}, \mathscr{H}\right)$,

(ii) $W(\Theta):=e^{\Theta A} W e^{-\Theta A}$ has an analytic continuation onto the strip $|\operatorname{Im} \Theta|<\alpha$.

Define $H(\kappa):=H+\kappa W$ on $D(H(\kappa))=\mathscr{H}_{2}$. Let $\lambda_{0}$ be an eigenvalue but no threshold of $H(0)$, let $P$ be the finite dimensional eigenprojection for $\lambda_{0}, \bar{P}:=I-P$, $\bar{H}(\kappa):=\bar{P} H(\kappa) \bar{P}$, and let $P W P$ have only simple eigenvalues.

Then Theorem 2.2 applies, that means we have resonances in the analytic sense, and in our sense.

Proof. Theorem 3.6 shows that $P(\Theta)=e^{\Theta A} P e^{-\Theta A}$ has an analytic continuation onto $|\operatorname{Im} \Theta|<\alpha$, hence so does $\bar{H}(\kappa, \Theta)=e^{\Theta A} \bar{H}(\kappa) e^{-\Theta A}$. Choose $\mathscr{H}_{+}=\mathscr{H}_{\beta}$ for some $\beta<\alpha$, then for $|\operatorname{Im} \Theta|<\beta$ and $\varphi, \psi \in \mathscr{H}_{+}$:

$$
\left.\left\langle\varphi,(\bar{H}(\kappa)-z)^{-1} \psi\right\rangle=\left\langle e^{\Theta A} \varphi, \bar{H}(\kappa, \Theta)-z\right)^{-1} e^{\Theta A} \psi\right\rangle .
$$

Using analytic perturbation theory, it is easily seen, that for sufficiently small $\kappa$ $\bar{H}(\kappa, \Theta)$ has no spectrum in a complex neighbourhood of $\lambda_{0}$. Hence $(\bar{H}(\kappa)-z)^{-1}$ has the required analytic continuation as an operator from $\mathscr{H}_{+}$to $\mathscr{H}_{-}$.

Assumption (ii) in 1.9 follows directly from Theorem 3.6, (iii) is redundant since after the above there are a priori no eigenvalues in the neighbourhoods considered, finally (iv) has been assumed.

Acknowledgements. The author would like to thank Joachim Weidmann, Florin Constantinescu and particularly Ulrich Scharffenberger for valuable discussions leading to the earlier german version of this work (the author's doctoral dissertation). Thanks also to Thomas Poerschke for his invaluable help in the preparation of the english version and to the referee for some good hints for streamlining the last section. 


\section{References}

[AC] Aguilar, J., Combes, J.M.: A class of analytic perturbations for one-body Schrödinger Hamiltonians. Commun. Math. Phys. 22, 269-279 (1971)

[BC] Balslev, E., Combes, J.M.: Spectral properties of many-body Schrödinger operators with dilation analytic interactions. Commun. Math. Phys. 22, 280-294 (1971)

[AHS] Agmon, S., Herbst, I., Skibsted, E.: Perturbation of embedded eigenvalues in the generalized $N$-body problem. Commun. Math. Phys. 122, 411-438 (1989)

[FH1] Froese, R., Herbst, I.: A new proof of the Mourré estimate. Duke. Math. J. 49, 1075-1085 (1982)

[FH2] Froese, R., Herbst, I.: Exponential bounds and absence of positive eigenvalues for $N$-body Schrödinger operators. Commun. Math. Phys. 87, 429-447 (1982/83)

[Her] Herbst, I.: Exponential decay in the Stark effect. Commun. Math. Phys. 75, 197-205 (1980)

[How] Howland, J.: The Livsic Matrix in perturbation theory. J. Math. Anal. Appl. 50, 415-437 (1975)

[JMP] Jensen, A., Mourré, E., Perry, P.: Multiple commutator estimates and resolvent smoothness in quantum scattering theory. Ann. Inst. Phys. Theor. 41, 253-261 (1984)

[Mo] Mourré, E.: Absence of singular continuous spectrum for certain self-adjoint operators. Commun. Math. Phys. 78, 391-408 (1981)

[Or] Orth, A.: Die mathematische Beschreibung von Resonanzen in VielteilchenQuantensystemen. Doctoral Dissertation Frankfurt 1985

[OP] Orth, A., Poerschke, T.: Quantum mechanical resonances and limiting absorption: the two body stark problem. Frankfurt University Preprint (1988)

[PSS] Perry, P., Sigal, I.M., Simon, B.: Spectral analysis of $N$-body Schrödinger operators. Ann. Math. 114, 519-567 (1981)

[RSIV] Reed, M., Simon, B.: Methods of modern mathematical physics. IV. Analysis of operators. New York: Academic Press 1978

[S1] Simon, B.: Resonances in $N$-body quantum systems with dilation-analytic potentials and the foundation of time-dependent perturbation theory. Ann. Math. 97, 247-274 (1973)

[S2] Simon, B.: Resonances and complex scaling: a rigorous overview. Int. J. Quantum Chem. 14, 529-542 (1978)

Communicated by T. Spencer

Received July 22, 1988; in revised form February 1, 1989 
